\title{
Trends and Social Barriers for Inpatient Palliative Care in Patients With Metastatic Bladder Cancer Receiving Critical Care Therapies
}

Elio Mazzone, MDa,b,c; Sophie Knipper, MDª,d; Francesco A. Mistretta, MDa,e; Carlotta Palumbo, MDa,f; Zhe Tian, PhDa Andrea Gallina, MD ${ }^{\mathrm{b}, \mathrm{c}}$; Derya Tilki, MDd,g; Shahrokh F. Shariat, MD, PhD; Francesco Montorsi, MD, FRCS b, ; Fred Saad, MD, FRCS ${ }^{a, i}$; Alberto Briganti, MD, PhD b,c; and Pierre I. Karakiewicz, MD ${ }^{a, i}$

\section{ABSTRACT}

Background: Use of inpatient palliative care (IPC) in the treatment of advanced cancer represents a well-established guideline recommendation. A recent analysis showed that patients with genitourinary cancer benefit from IPC at the second lowest rate among 4 examined primary cancers, namely lung, breast, colorectal, and genitourinary. Based on this observation, temporal trends and predictors of IPC use were examined in patients with metastatic urothelial carcinoma of the bladder (mUCB) receiving critical care therapies (CCTs). Patients and Methods: Patients with mUCB receiving CCTs were identified within the Nationwide Inpatient Sample database (2004-2015). IPC use rates were evaluated in estimated annual percentage change (EAPC) analyses. Multivariable logistic regression models with adjustment for clustering at the hospital level were used. Results: Of 1,944 patients with $\mathrm{mUCB}$ receiving CCTs, 191 (9.8\%) received IPC. From 2004 through 2015, IPC use increased from $0.7 \%$ to $25.0 \%$, respectively (EAPC, $+23.9 \% ; P<.001$ ). In analyses stratified according to regions, the highest increase in IPC use was recorded in the Northeast (EAPC, $+44.0 \%$ ), followed by the West (EAPC, +26.8\%), South (EAPC, $+22.9 \%)$, and Midwest (EAPC, $+15.5 \%)$. Moreover, the lowest rate of IPC adoption in 2015 was recorded in the Midwest (14.3\%). In multivariable logistic regression models, teaching status (odds ratio [OR], 1.97; $P<.001$ ), more recent diagnosis (2010-2015; OR, 3.89; $P<.001)$, and presence of liver metastases (OR, 1.77; $P=.02$ ) were associated with higher IPC rates. Conversely, Hispanic race $(O R, 0.42 ; P=.03)$ and being hospitalized in the Northeast $(O R, 0.36$; $P=.01)$ were associated with lower rate of IPC adoption. Finally, patients with a primary admission diagnosis that consisted of infection (OR, 2.05; $P=.002)$, cardiovascular disorders $(O R, 2.10 ; P=.03)$, or pulmonary disorders $(O R, 2.81 ; P=.005)$ were more likely to receive IPC. Conclusions: The rate of IPC use in patients with $\mathrm{mUCB}$ receiving CCTs sharply increased between 2004 and 2015. The presence of liver metastases, infections, or cardiopulmonary disorders as admission diagnoses represented independent predictors of higher IPC use. Conversely, Hispanic race, nonteaching hospital status, and hospitalization in the Midwest were identified as independent predictors of lower IPC use and represent targets for efforts to improve IPC delivery in patients with $\mathrm{mUCB}$ receiving CCT.

J Natl Compr Canc Netw 2019;17(11):1344-1352 doi: 10.6004/jnccn.2019.7319

\section{Background}

The integration of palliative care into the treatment of advanced cancer represents an established guideline recommendation ${ }^{1-3}$ and has been considered an indicator of quality of care. ${ }^{4-11}$ It is noteworthy that early introduction of palliative care may improve not only quality of life but also survival in patients with advanced cancer, ${ }^{11}$ and may reduce length of hospital stay and cost. ${ }^{12,13}$ Therefore, equal access to palliative care should be warranted in all patients with metastatic cancer, especially those requiring critical care therapies (CCTs) for treatment-related complications or disease progression.

A recent publication focusing on patients from California during 2010 showed the integration of inpatient palliative care (IPC) in patients receiving CCTs across a variety of metastatic tumor primaries, including genitourinary (GU) cancer. ${ }^{14}$ In that report, patients with GU cancer benefited from IPC at the second lowest rate among 4 examined primary cancers (lung, breast, colorectal, and GU). ${ }^{14}$ Based on this finding, temporal trends in and social barriers to IPC delivery in patients receiving CCTs were examined in a larger national patient sample and across time. Within the GU category, we focused on metastatic urothelial carcinoma of the bladder (mUCB) because it is associated with poor prognosis, even in contemporary analyses. ${ }^{15,16}$ We hypothesized that, despite occupying the second lowest

${ }^{a}$ Cancer Prognostics and Health Outcomes Unit, University of Montreal Health Center, Montreal, Quebec, Canada; ${ }^{b}$ Division of Experimental Oncology, Unit of Urology, Urological Research Institute, IRCCS San Raffaele Scientific Institute, and 'Vita-Salute San Raffaele University, Milan, Italy; ${ }^{\mathrm{d}}$ Martini Klinik, University Medical Center Hamburg-Eppendorf, Hamburg, Germany; ${ }^{e}$ Department of Urology, European Institute of Oncology, Milan, Italy; ${ }^{f}$ Department of Urology, Spedali Civili Hospital, University of Brescia, Brescia, Italy; ${ }^{9}$ Department of Urology, University Medical Center Hamburg-Eppendorf, Hamburg, Germany; hDepartment of Urology, Medical University of Vienna, Vienna, Austria; and 'Division of Urology, University of Montreal Hospital Center, Montreal, Quebec, Canada. 
position among other primaries, IPC use rate improved over time in patients with mUCB receiving CCTs. In addition, we tested for potential barriers to IPC delivery in these patients when CCT is delivered.

\section{Patients and Methods}

Data Source and Patient Selection

We relied on the Nationwide Inpatient Sample (NIS) database (2004-2015) to assess trends in and predictors of IPC use in patients receiving CCTs. The NIS is a set of longitudinal hospital inpatient databases included in the Healthcare Cost and Utilization Project family, created by the Agency for Healthcare Research and Quality (AHRQ) through a federal-state partnership. ${ }^{17}$ The database includes $20 \%$ of US inpatient hospitalizations with discharge abstracts from 8 million hospital stays.

\section{Study Population}

We focused on patients aged $\geq 18$ years with a diagnosis of mUCB (ICD-9-CM code 188.0) who received CCTs. All procedures and diagnoses were coded using ICD9-CM. Procedure codes were used to identify CCTs, specifically invasive mechanical ventilation (ICD-9-CM codes 96.70-96.72), percutaneous endoscopic gastrostomy tube (ICD-9-CM code 43.11), dialysis for acute kidney failure (ICD-9-CM codes 584.5-585.9, and 39.95 to exclude patients receiving chronic dialysis), total parenteral nutrition (ICD-9-CM code 99.15), and tracheostomy (ICD-9-CM codes 31.1, 31.21, 31.29). ${ }^{14,18}$ Only patients with a secondary diagnosis of metastatic disease were included (ICD-9-CM codes 197.x and 198.x). These selection criteria yielded 1,944 patients.

\section{Patient and Hospital Characteristics}

Patient age at admission, year of admission, sex, race/ ethnicity (white, African American, Hispanic, and other/ unknown), Charlson comorbidity index score, ${ }^{19,20}$ and insurance status (private, Medicare, Medicaid, and other [self-pay]) were ascertained from the NIS. For purposes of univariable and multivariable analyses, year of admission was dichotomized in intervals, namely historical (2004-2009) and contemporary (2010-2015). Additional risk variables consisted of hospital region (Northeast, Midwest, South, West), hospital size (small, $<200$ beds; medium, 200-399 beds; large, $\geq 400$ beds), and hospital teaching versus nonteaching status. Teaching institutions had an American Medical Association-approved residency program, were members of the Council of Teaching Hospitals and Health Systems, or had a ratio of $\geq 0.25$ of fulltime equivalent interns and residents to non-nursing home beds. ${ }^{21}$ Last, inflation-adjusted total hospital charges were defined according to NIS methodology. ${ }^{17}$
Definition of Principal Diagnosis and IPC Use We classified the principal diagnosis using the Clinical Classifications Software codes. Clinical Classifications Software is a classification tool developed by the AHRQ that collapses $>14,000$ ICD-9-CM diagnosis codes and 3,900 ICD-9-CM procedure codes into a smaller set of clinically meaningful categories. ${ }^{14,17,18}$ Use of IPC services was defined by the ICD-9 diagnosis code V66.722-24; two prior validation studies showed that this code for identification of IPC services in patients with metastatic disease has a sensitivity of $66.3 \%$ to $84.0 \%$ and specificity of $95.0 \%$ to $99.1 \% .^{22,23}$ Only hospitals that provided at least one IPC service were included. Therefore, all of the hospitals included in the analysis had access to IPC.

\section{Statistical Analysis and Covariates}

To assess contemporary use rates and disparities in IPC use, 3 analytical steps were followed. First, medians with interquartile ranges and frequencies with proportions were reported for continuous and categorical variables, respectively, and the statistical significance of differences was evaluated using Kruskal-Wallis and chi-square tests. Second, estimated annual percentage change (EAPC) was defined in the overall cohort and in subgroup analyses focusing on regions and hospital teaching status. Third, multivariable logistic regression (MLR) models were used to test for predictors of access to IPC services. All multivariable analyses were fitted after adjustment for clustering at the hospital level using generalized estimation equation methodology. ${ }^{25}$ In MLR models, adjustment variables consisted of age at admission, year of admission, sex, race, Charlson comorbidity index score, hospital teaching status, hospital region, insurance status, hospital size, metastatic sites, primary diagnosis at admission, and type of CCT.

All statistical tests were 2-sided with the level of significance set at $P<.05$. Analyses were performed using R software (version 3.5.1).

\section{Results}

\section{Study Population}

Between 2004 and 2015, 1,944 patients with mUCB received CCT; of those, 191 (9.8\%) received IPC (Table 1). Mean total hospital charges among survivors (ie, patients discharged alive at the end of the hospital stay) were lower in patients who received IPC than in those who did not ( $\$ 35,524$ vs $\$ 45,047 ; P=.04$ ). Similarly, length of stay among survivors was shorter in patients who received IPC than in those who did not ( 11 vs 13 days; $P=.002$ ). IPC services were more frequently delivered to white patients ( $76.4 \%$ vs $70.1 \%$; $P=.06)$, in teaching hospitals $(68.1 \%$ vs 57.4\%; $P=.005$ ), and to patients with liver metastases ( $20.4 \%$ vs $10.0 \% ; P<.001$ ). According to principal diagnosis 
Table 1. Baseline Patient Characteristics

\begin{tabular}{|c|c|c|c|c|}
\hline Variable & Overall & IPC & No IPC & $P$ Value ${ }^{a}$ \\
\hline Total, n (\%) & 1,944 & $191(9.8 \%)$ & $1,753(90.2 \%)$ & \\
\hline Age at admission, y & & & & .1 \\
\hline Median & 71 & 70 & 71 & \\
\hline IQR & $62-78$ & $59-78$ & $63-78$ & \\
\hline LOS (only survivors), d & & & & .002 \\
\hline Median & 13 & 11 & 13 & \\
\hline IQR & $8-20$ & $6-18$ & $8-20$ & \\
\hline THCs (only survivors), mean (SD), \$USD & $\$ 44,359(\$ 41,330)$ & $\$ 35,524(\$ 44,440)$ & $\$ 45,047(\$ 41,016)$ & .04 \\
\hline Year interval, n (\%) & & & & $<.001$ \\
\hline 2004-2009 & $947(48.7)$ & $37(19.4)$ & $910(51.9)$ & \\
\hline 2010-2015 & $997(51.3)$ & $154(80.6)$ & $843(48.1)$ & \\
\hline Sex, n (\%) & & & & .3 \\
\hline Male & $1,468(75.5)$ & $138(72.3)$ & $1,330(75.9)$ & \\
\hline Female & $476(24.5)$ & $53(27.7)$ & $423(24.1)$ & \\
\hline Race, n (\%) & & & & .06 \\
\hline White & $1,374(70.7)$ & $146(76.4)$ & $1,228(70.1)$ & \\
\hline African American & $195(10.0)$ & $22(11.5)$ & $173(9.9)$ & \\
\hline Hispanic & $99(5.1)$ & $7(3.7)$ & $92(5.2)$ & \\
\hline Other/Unknown & $276(14.2)$ & $16(8.4)$ & $260(14.8)$ & \\
\hline Charlson comorbidity index score, $\mathrm{n}(\%)$ & & & & .4 \\
\hline 0 & $1,121(57.7)$ & $115(60.2)$ & $1,006(57.4)$ & \\
\hline 1 & $486(25.0)$ & $40(20.9)$ & $446(25.4)$ & \\
\hline$\geq 2$ & $337(17.3)$ & $36(18.8)$ & $301(17.2)$ & \\
\hline Hospital teaching status, n (\%) & & & & .005 \\
\hline Nonteaching & $808(41.6)$ & $61(31.9)$ & $747(42.6)$ & \\
\hline Teaching & $1,136(58.4)$ & $130(68.1)$ & $1,006(57.4)$ & \\
\hline Hospital region, n (\%) & & & & .08 \\
\hline South & $634(32.6)$ & $72(37.7)$ & $562(32.1)$ & \\
\hline Midwest & $442(22.7)$ & $42(22.0)$ & $400(22.8)$ & \\
\hline Northeast & $461(23.7)$ & $32(16.8)$ & $429(24.5)$ & \\
\hline West & $407(20.9)$ & $45(23.6)$ & $362(20.7)$ & \\
\hline Insurance status, n (\%) & & & & .08 \\
\hline Medicare & $1,288(66.3)$ & $121(63.4)$ & $1,167(66.6)$ & \\
\hline Medicaid & $161(8.3)$ & $25(13.1)$ & $136(7.8)$ & \\
\hline Private & $418(21.5)$ & $39(20.4)$ & $379(21.6)$ & \\
\hline Other & $77(4.0)$ & $6(3.1)$ & $71(4.1)$ & \\
\hline Hospital size, ${ }^{\mathrm{b}}$ n (\%) & & & & .5 \\
\hline Large ( $\geq 400$ beds) & $1,263(65.0)$ & $117(61.3)$ & $1,146(65.4)$ & \\
\hline Medium (200-399 beds) & $448(23.0)$ & $48(25.1)$ & $400(22.8)$ & \\
\hline Small (<200 beds) & $233(12.0)$ & $26(13.6)$ & 207 (11.8) & \\
\hline
\end{tabular}




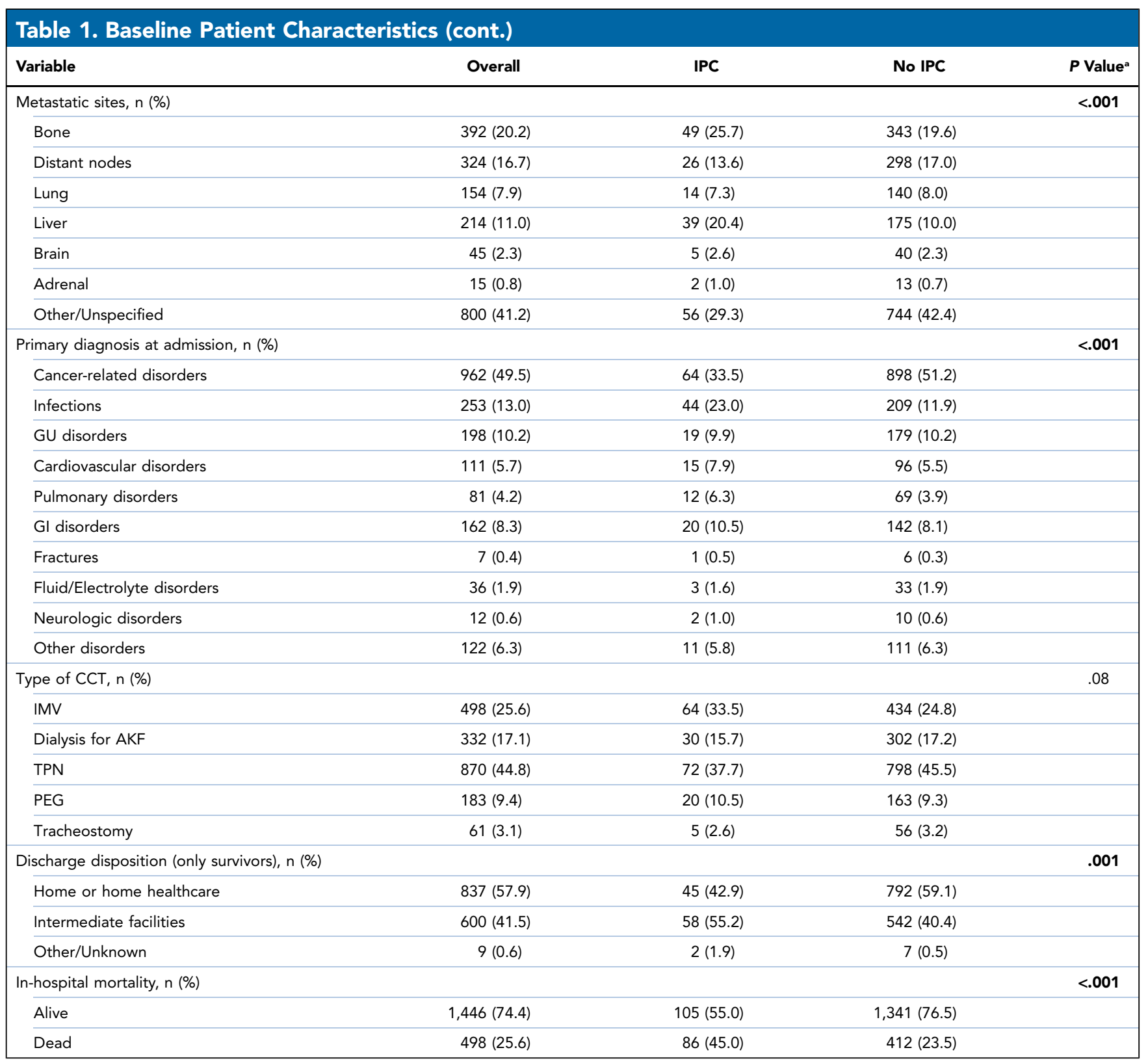

Abbreviations: AKF, acute kidney failure; CCT, critical care therapy; GI, gastrointestinal; GU, genitourinary; IMV, invasive mechanical ventilation; IPC, inpatient palliative care; IQR, interquartile range; LOS, length of stay; PEG, percutaneous endoscopic gastrostomy; THCs, total hospital charges; TPN, total parenteral nutrition.

aBold indicates statistically significant $P$ value.

bBased on number of beds.

at admission, IPC services were more frequently used in patients admitted for infections $(23.0 \%$ vs $11.9 \%$; $P<.001$ ), cardiovascular disorders ( $7.9 \%$ vs $5.5 \% ; P<.001$ ), or pulmonary disorders $(6.3 \%$ vs $3.9 \% ; P<.001)$. Conversely, IPC services were less frequently used in patients admitted for cancer-related disorders $(33.5 \%$ vs $51.2 \%$; $P<.001)$. Finally, in-hospital mortality was higher in patients who received IPC than in those who did not (45.0\% vs $23.5 \%$; $P<.001$ ) (Table 1 ).

After stratification according to regions, the highest overall rate of IPC use was recorded in the South
(11.4\%), followed by the West (11.0\%), the Midwest (9.5\%), and the Northeast (6.9\%). Similarly, after stratification according to teaching status, the overall rate of IPC use was higher in teaching hospitals (11.4\%) than in nonteaching hospitals (7.5\%).

\section{Trends of IPC Use}

Overall, IPC use rates in patients with mUCB receiving CCTs increased from $0.7 \%$ in 2004 to $25.0 \%$ in 2015 (EAPC, $+23.9 \%$; 95\% CI, +18.5 to $+30.4 ; P<.001$ ) (Figure 1 ). In subgroup analyses stratified according to regions, IPC 


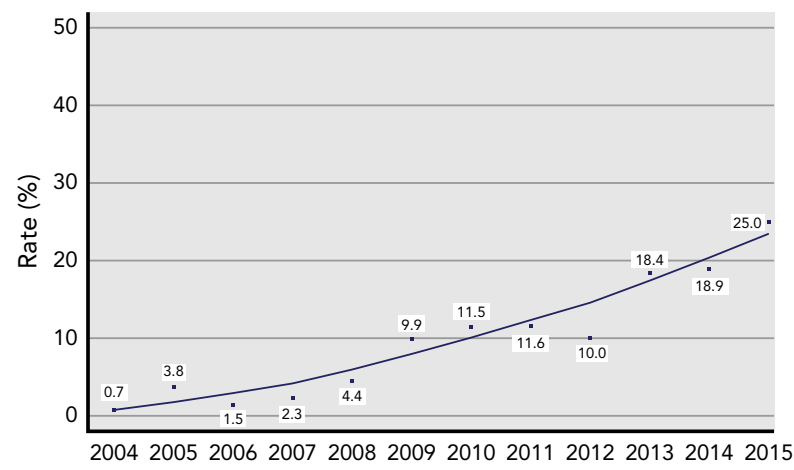

Figure 1. Inpatient palliative care delivery rate over time.

use rate increased from $2.6 \%$ to $24.1 \%$ in the South (EAPC, $+22.9 \%$; $95 \% \mathrm{CI},+14.9 \%$ to $+33.9 \%$; $P<.001$ ), from $0 \%$ to $34.6 \%$ in the West (EAPC, $+26.8 \%$; $95 \% \mathrm{CI},+11.9 \%$ to $+59.0 \%$; $P=.006$ ), from $0 \%$ to $14.3 \%$ in the Midwest (EAPC, $+15.5 \%$; $95 \% \mathrm{CI},+7.8 \%$ to $+25.8 \% ; P=.007$ ), and from $0 \%$ to $26.1 \%$ in the Northeast (EAPC, $+44.0 \%$; $95 \%$ CI, $+29.2 \%$ to $+68.7 \% ; P<.001$ ) (Figure 2). In additional subgroup analyses stratified by teaching status, IPC use rate increased from $0 \%$ to $25.8 \%$ in teaching hospitals (EAPC,

A

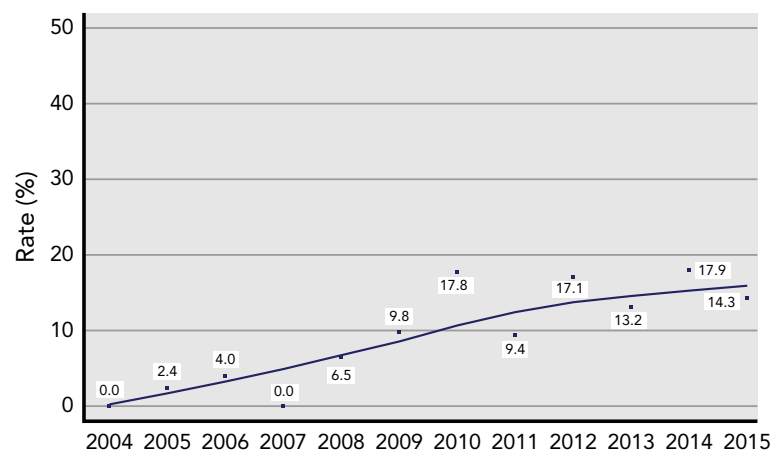

C

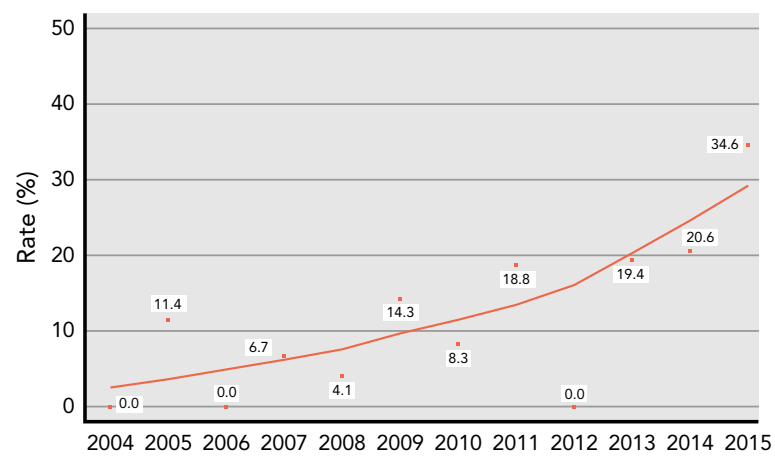

$+25.0 \%$; $95 \%$ CI, $+19.4 \%$ to $+32.0 \% ; P<.001)$ and from $1.4 \%$ to $23.1 \%$ in nonteaching hospitals (EAPC, $+18.9 \%$; $95 \% \mathrm{CI},+9.6 \%$ to $+38.4 \%$; $P=.009$ ) (Figure 3 ).

\section{MLR Models Predicting Use of IPC}

In MLR models adjusted for clustering and all available covariates, hospital teaching status (odds ratio [OR], 1.97; 95\% CI, 1.37-2.85; $P<.001$ ), contemporary years interval (2010-2015) (OR, 3.89; 95\% CI, 2.63-5.77; $P<.001$ ), and presence of liver metastases (OR, 1.77; 95\% CI, 1.09-2.88; $P=.02$ ) were associated with higher IPC use in patients with mUCB receiving CCT relative to nonteaching status, historical years interval (2004-2009), and presence of bone metastases, respectively (Table 2). Conversely, Hispanic race (OR, 0.42; 95\% CI, 0.18-0.95; $P=.03$ ) and being hospitalized in the Northeast $(\mathrm{OR}, 0.36$; $95 \% \mathrm{CI}, 0.21-0.87 ; P<.001)$ were associated with lower IPC use than white race and being hospitalized in the West, respectively.

Regarding primary diagnosis at admission, patients with a primary diagnosis of infections (OR, 2.05; 95\% CI, 1.29-3.26; $P=.002)$, cardiovascular disorders (OR, 2.10; 95\% CI, 1.04-4.22; $P=.03$ ), or pulmonary disorders (OR, 2.81; 95\% CI, 1.35-5.86; $P=.005$ ) were more likely than patients with cancer-related disorders to receive

\section{B}

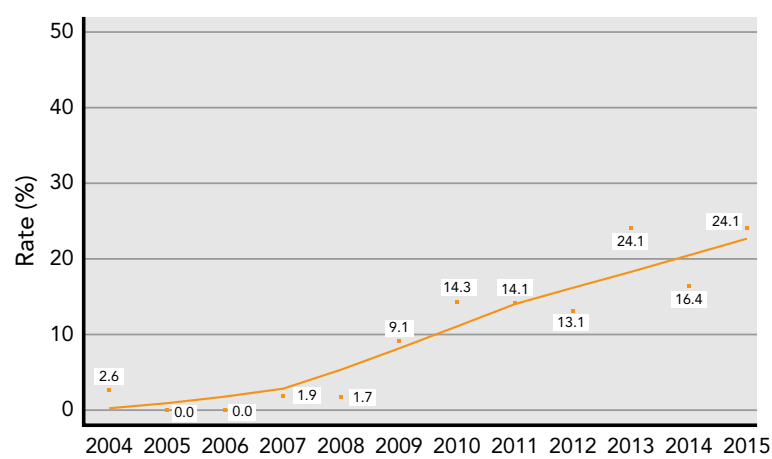

D

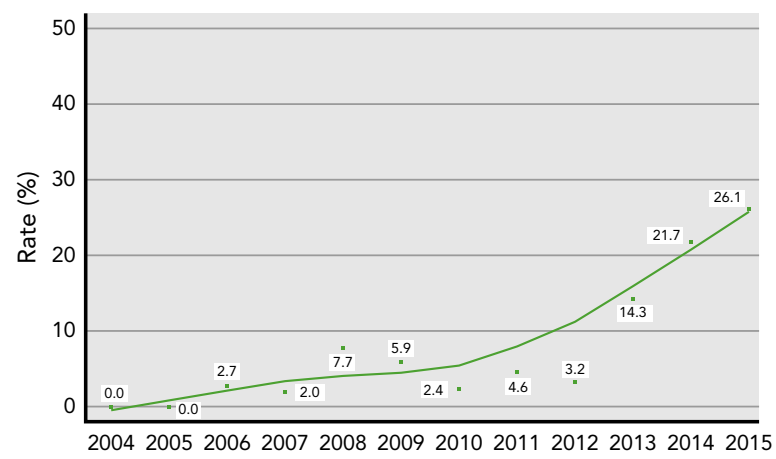

Figure 2. Inpatient palliative care delivery rate over time, stratified according to geographic region: (A) Midwest, (B) South, (C) West, and (D) Northeast. 
A

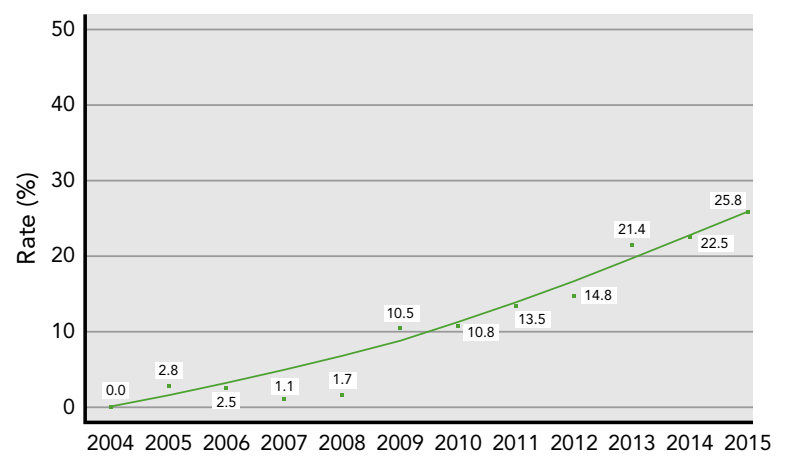

B

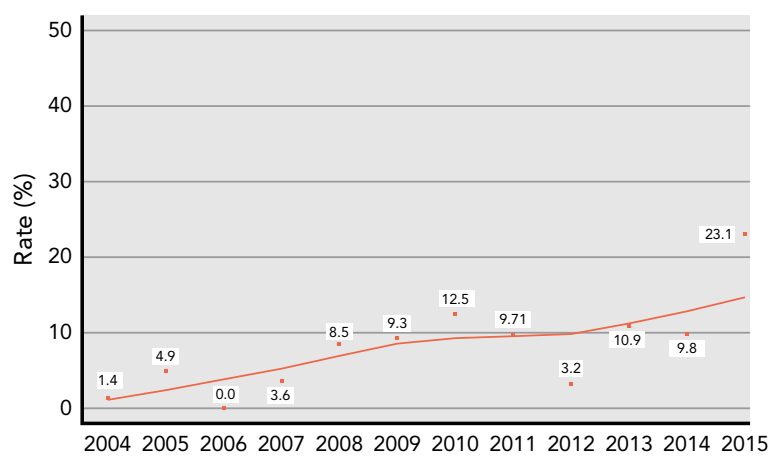

Figure 3. Inpatient palliative care delivery rate over time, stratified according to hospital (A) teaching and (B) nonteaching status.

IPC. Finally, regarding CCT types, no differences in IPC administration were recorded (Table 2).

\section{Discussion}

IPC delivery in patients with advanced cancer represents a well-established guideline recommendation. ${ }^{1-3}$ However, in a previous report by Loh et al, ${ }^{14}$ the rate of IPC use was among the lowest in patients with metastatic GU cancer receiving CCTs. This observation is worrisome and may indicate suboptimal quality of care in patients with advanced GU malignancies, especially those with a poor prognosis, such as patients with mUCB. Based on this concept, we hypothesized that IPC use increases over time in patients with mUCB receiving CCTs. To address this, we examined the use of IPC in the NIS over a period of 12 years (2004-2015) and also tested for potential predictors of IPC use. These analyses revealed several noteworthy findings.

First, the overall IPC use rate was low at $9.8 \%$. However, the annual rate sharply increased from $0.7 \%$ to $25.0 \%$ in a highly statistically significant fashion during the study period (EAPC, $+23.9 \%$; $P<.001$ ). This observation suggests improved adherence to North American guidelines and, most important, improved patient care. The previously reported $16.8 \%$ rate of IPC use among patients with GU cancer recorded in California in 2010 was higher than the national average of $9.8 \%$ recorded in our study among patients with mUCB between 2004 and $2015 .{ }^{14}$ Thus, the reference standard reported by Loh et $\mathrm{al}^{14}$ originated from a state in which the rates of IPC use and possibly the quality of care for this specific patient population are higher than the national average. Indeed, the highest rate of IPC use in 2015 was recorded in the West (34.6\%), which includes California. Despite the enthusiasm related to an important absolute and relative increase in IPC delivery across time, our analyses of IPC delivery rates in patients with mUCB receiving CCTs showed a maximum national overall rate of $25.0 \%$ in 2015.
This rate is still lower than the highest individual rate of IPC use (28.3\%) reported among patients with lung cancer in 2010 within the California state analysis. Therefore, further efforts aimed at educating and sensitizing clinicians involved in the critical care of patients with metastatic prostate cancer nationwide are still needed.

Second, we reported differences in trends over time in the 4 examined geographic regions. Despite the lowest overall rate of IPC use being recorded in the Northeast in both univariable and multivariable analyses, we showed that IPC use sharply increased in more recent years in this region, as shown by the highest EAPC $(+44.4 \%)$ recorded there among all 4 examined regions. Therefore, this pronounced increasing trend recorded in the Northeast may be interpreted as the increasing adoption of IPC use more recently than in other regions, such as the South or West. Conversely, the lowest IPC use increase and the lowest overall IPC use rate were recorded in the Midwest (EAPC, $+15.5 \%$ ). Taken together, these differences imply that efforts aimed at improving IPC rate delivery may need to be of greater intensity in specific regions, such as the Midwest, where the rate of IPC adoption over time was low.

Third, we also recorded an important effect of race on the rate of IPC delivery. Specifically, Hispanic patients were significantly less likely to receive IPC, as shown in MLR models (hazard ratio, 0.42; $P=.03$ ). To the best of our knowledge, we are the first to record disparities in access to end-of-life care among patients with mUCB, and therefore a direct comparison with previous reports cannot be performed. However, racial disparities in access to end-of-life care were identified in other metastatic primaries, such as prostate ${ }^{26}$ and lung cancer. ${ }^{27}$

Fourth, we identified hospital teaching status as an important predictor of IPC delivery. IPC delivery rates were higher across teaching hospitals in both univariable $(11.4 \%$ vs $7.5 \% ; P<.001)$ and multivariable analyses (OR, 1.97; $P<.001$ ). This difference was persistent across the study 


\begin{tabular}{|c|c|c|}
\hline Variable & OR $(95 \% \mathrm{Cl})$ & P Value ${ }^{a}$ \\
\hline \multicolumn{3}{|l|}{ Teaching status } \\
\hline Nonteaching & Ref & \\
\hline Teaching & $1.97(1.37-2.85)$ & $<.001$ \\
\hline \multicolumn{3}{|l|}{ Year interval } \\
\hline 2004-2009 & Ref & \\
\hline 2010-2015 & $3.89(2.63-5.77)$ & $<.001$ \\
\hline \multicolumn{3}{|l|}{ Race } \\
\hline White & Ref & \\
\hline African American & $0.78(0.46-1.33)$ & .4 \\
\hline Hispanic & $0.42(0.18-0.95)$ & .03 \\
\hline Other/Unknown & $0.52(0.32-0.83)$ & .007 \\
\hline \multicolumn{3}{|l|}{ Hospital region } \\
\hline West & Ref & \\
\hline Midwest & $0.62(0.38-1.03)$ & .07 \\
\hline Northeast & $0.36(0.21-0.62)$ & $<.001$ \\
\hline South & $0.66(0.42-1.03)$ & .07 \\
\hline \multicolumn{3}{|l|}{ Metastatic site } \\
\hline Bone & Ref & \\
\hline Distant nodes & $0.60(0.34-1.04)$ & .07 \\
\hline Lung & $0.64(0.32-1.28)$ & .2 \\
\hline Liver & $1.77(1.09-2.88)$ & .02 \\
\hline Brain & $0.71(0.27-1.85)$ & .4 \\
\hline Adrenal & $1.21(0.20-7.30)$ & .8 \\
\hline Other/Unspecified & $0.62(0.39-0.98)$ & .04 \\
\hline \multicolumn{3}{|l|}{ Type of CCT } \\
\hline IMV & Ref & \\
\hline Dialysis for AKF & $0.71(0.41-1.21)$ & .2 \\
\hline TPN & $0.87(0.58-1.32)$ & .5 \\
\hline PEG & $1.05(0.58-1.90)$ & .8 \\
\hline Tracheostomy & $0.62(0.26-1.47)$ & .2 \\
\hline \multicolumn{3}{|l|}{ Primary diagnosis } \\
\hline Cancer-related disorders & Ref & \\
\hline Infections & $2.05(1.29-3.26)$ & .002 \\
\hline GU disorders & $1.61(0.86-3.00)$ & .1 \\
\hline Cardiovascular disorders & $2.10(1.04-4.22)$ & .03 \\
\hline Pulmonary disorders & $2.81(1.35-5.86)$ & .005 \\
\hline GI disorders & $1.67(0.92-3.03)$ & .08 \\
\hline Fractures & $2.14(0.22-20.90)$ & .5 \\
\hline Fluid/Electrolyte disorders & $1.38(0.40-4.67)$ & .6 \\
\hline Neurologic disorders & $3.15(0.76-13.07)$ & .1 \\
\hline Other disorders & $1.25(0.61-2.55)$ & .5 \\
\hline
\end{tabular}

Model was adjusted for Charlson comorbidity index score, age at admission, sex, insurance status, and hospital size (number of beds).

Abbreviations: AKF, acute kidney failure; $\mathrm{CCT}$, critical care therapy; $\mathrm{Gl}$,

gastrointestinal; GU, genitourinary; IMV, invasive mechanical ventilation; OR, odds ratio; PEG, percutaneous endoscopic gastrostomy; TPN, total parenteral nutrition. aBold indicates statistically significant $P$ value. period. Specifically, IPC use rates increased, with EAPCs of $+25.0 \%$ in teaching hospitals and $+18.9 \%$ in nonteaching hospitals. This association between nonteaching hospitals and lower IPC rates was not identified in California, ${ }^{14}$ which may be explained by overall higher rates of IPC delivery in California. Moreover, specific analyses that focused on IPC delivery according to teaching status were not performed in the subgroup of GU cancers in the analysis by Loh et al. ${ }^{14}$

Fifth, IPC use in patients with mUCB receiving CCTs was significantly associated with the variables defining metastatic sites and primary diagnosis at admission. Specifically, patients with liver metastases were more likely than those with bone metastases to receive IPC services (OR, 1.77; $P=.02)$. This finding may be interpreted as higher IPC use in patients with poor prognosis, because liver metastases have been shown to be associated with the poorest prognosis in patients with mUCB. ${ }^{28}$ Similarly, patients whose primary diagnosis at admission consisted of infections (OR, 2.05; $P=.002)$, cardiovascular disorders (OR, $2.10 ; P=.03)$, or pulmonary disorders (OR, 2.81; $P=.005)$ received IPC at a higher rate than those with cancer-related disorders. The higher rate of IPC use in patients presenting with infections, cardiovascular disorders, or pulmonary disorders as their primary diagnosis at admission (eg, acute myocardial infarction or acute respiratory distress syndrome) can be explained by the highly life-threatening nature of these complaints relative to patients admitted for disorders directly related to cancer, in whom the gravity of individual diagnoses may vary. Our results are in agreement with those of Loh et $\mathrm{al}^{14}{ }^{14}$ who reported that a higher probability of receiving IPC services was recorded in patients with a primary diagnosis of infection. However, Loh et $\mathrm{al}^{14}$ did not perform specific subgroup analyses focusing on patients with GU cancer, and therefore valid conclusions about agreement or disagreement between these studies cannot be drawn.

Taken together, our findings showed important absolute and relative increases in IPC delivery among patients with mUCB receiving CCT. However, after stratification according to geographic region, we showed lower uptake of IPC use in the Midwest. Moreover, we identified Hispanic race and nonteaching hospital status as barriers to IPC delivery. Additionally, the presence at admission of specific underlying conditions that have highly life-threatening implications, namely liver metastases, infection, cardiovascular disorders, or pulmonary disorders, was identified as a predictor of higher IPC use rate. These observations indicate that, despite improvements, there is a persistent need for efforts aimed at IPC-focused education and sensitization that should particularly target healthcare providers of Hispanic patients, at nonteaching institutions, and in the Midwest. Moreover, it should be noted that, relative to the study by Loh et al, ${ }^{14}$ our study was based on an exponentially larger 
patient sample and allowed for a specifically focused analysis of patients with mUCB in addition to temporal analyses and analyses according to the 4 geographic regions. Last, it is also important to note that all multivariable models were performed with additional adjustment for clustering at the hospital level using a generalized estimation equation. ${ }^{29,30}$ This methodology accurately accounted for interhospital variability in IPC use that may have determined the recorded differences in access to IPC. Therefore, our analysis was not affected by residual biases related to severe underuse of IPC services at some institutions.

The decision to use the V66.7 ICD-9 code to discern IPC services was based on recent and solid validation studies. ${ }^{22,23,31}$ Specifically, Feder et $\mathrm{al}^{22}$ performed the largest validation study of this code using the Veterans Health Administration database. They recorded high sensitivity $(84 \%)$ and very high specificity $(98 \%)$ that confirm the reliability of this code for IPC use. In consequence, from a clinical standpoint, it is unlikely that patients who received IPC were misclassified. Moreover, the study by Feder et $\mathrm{a}^{22}$ represents the most recent validation of the V66.7 code and largely outperformed previous validation studies in terms of sensitivity ( $84 \%$ vs $66 \%$ ). ${ }^{22,23,31}$ This finding can also be interpreted as indicative of increasing confidence among clinicians regarding application of this code. Taken together, this evidence underlines the solidity of the ICD-9 code for identifying IPC services and confirms the validity of its use in our analysis. However, these differences in code sensitivity may also reflect variations in billing and coding practices across health systems and the coding input for their respective administrative datasets. Therefore, some residual confounding factors may have remained operational in our analysis.

Our study is not devoid of limitations. First, as is true of previous analyses of IPC use in other cancer databases, ${ }^{14,26}$ our study is based on a retrospective analysis with all of its inherent limitations. Second, we only included specific CCTs, and our findings therefore do not apply to other types of CCTs. Third, IPC was defined according to the V66.7 ICD-9 code, which is characterized by a sensitivity ranging from $66 \%$ to $84 \% .{ }^{22,23}$ Therefore, the number of patients who used IPC services may be underestimated. Fourth, we were also unable to adjust for patient characteristics that may have contributed to reported differences in IPC use rates, such as performance status; American Society of Anesthesiologists physical status; differences in laboratory values and opioid use; and presence or absence of exposure to chemotherapy, androgen deprivation, or radiotherapy. Fifth, the NIS database does not allow identification of the exact time when patients were referred for IPC services during their hospital stay. Similarly, we cannot code sequences of interventions or explore the correlation between use of IPC and escalation in procedural intervention. In addition, only inpatient data are available in the NIS database. Thus, we could not examine postdischarge management. These factors should ideally be included when analyses focusing on IPC use in advanced cancers are performed.

\section{Conclusions}

IPC use in patients with mUCB receiving CCTs sharply increased between 2004 and 2015. The presence of liver metastases and infections or cardiopulmonary disorders at admission were independent predictors of higher IPC use. Conversely, Hispanic race, nonteaching hospital status, and hospital location in the Midwest were identified as independent predictors of lower IPC use and represent targets for efforts aimed at improving IPC delivery in patients with mUCB receiving CCTs.

Submitted November 2, 2018; accepted for publication May 15, 2019.

Author contributions: Study concept and design: Mazzone, Knipper, Karakiewicz. Data curation: Mistretta, Palumbo. Formal analysis: Mazzone, Mistretta, Tian. Methodology: Mazzone, Tian, Gallina, Tilki. Project administration: Tian, Montorsi, Karakiewicz. Software: Tian. Supervision: Shariat, Montorsi, Saad, Karakiewicz. Visualization: Mazzone, Knipper, Tian. Writing of original draft: Mazzone, Karakiewicz. Manuscript review and editing: Montorsi, Briganti.

Disclosures: The authors have not received any financial consideration from any person or organization to support the preparation, analysis, results, or discussion of this article.

Correspondence: Elio Mazzone, MD, Division of Experimental Oncology, Unit of Urology, Urological Research Institute, IRCCS San Raffaele Scientific Institute, Via Olgettina 60, Milan 20132, Italy. E-mail: eliomazzone@gmail.com

\section{References}

1. Morrison RS, Meier DE. Palliative care. N Engl J Med 2004;350:2582-2590.

2. Ferrell BR, Temel JS, Temin S, et al. Integration of palliative care into standard oncology care: American Society of Clinical Oncology clinical practice guideline update. J Clin Oncol 2017;35:96-112.

3. Smith TJ, Temin S, Alesi ER, et al. American Society of Clinical Oncology provisional clinical opinion: the integration of palliative care into standard oncology care. J Clin Oncol 2012;30:880-887.

4. Connors AF Jr, Dawson NV, Dsbiens NA, et al. A controlled trial to improve care for seriously ill hospitalized patients: the Study to Understand Prognoses and Preferences for Outcomes and Risks of Treatments (SUPPORT). JAMA 1995;274:1591-1598.

5. Malin JL. Bridging the divide: integrating cancer-directed therapy and palliative care. J Clin Oncol 2004;22:3438-3440.

6. Greer JA, Jackson VA, Meier DE, et al. Early integration of palliative care services with standard oncology care for patients with advanced cancer. CA Cancer J Clin 2013;63:349-363.

7. Meyers FJ, Linder J. Simultaneous care: disease treatment and palliative care throughout illness. J Clin Oncol 2003;21:1412-1415.

8. Davis MP, Bruera E, Morganstern D. Early integration of palliative and supportive care in the cancer continuum: challenges and opportunities. Am Soc Clin Oncol Educ Book 2013:144-150.

9. Abrahm JL. Integrating palliative care into comprehensive cancer care. J Natl Compr Canc Netw 2012;10:1192-1198.

10. Temel JS, Greer JA, El-Jawahri A, et al. Effects of early integrated palliative care in patients with lung and GI cancer: a randomized clinical trial. $\mathrm{J}$ Clin Oncol 2017;35:834-841. 
11. Temel JS, Greer JA, Muzikansky A, et al. Early palliative care for patients with metastatic non-small-cell lung cancer. N Engl J Med 2010;363: 733-742.

12. Khandelwal N, Kross EK, Engelberg RA, et al. Estimating the effect of palliative care interventions and advance care planning on ICU utilization: a systematic review. Crit Care Med 2015;43:1102-1111.

13. May P, Garrido MM, Cassel JB, et al. Prospective cohort study of hospital palliative care teams for inpatients with advanced cancer: earlier consultation is associated with larger cost-saving effect. J Clin Oncol 2015;33: 2745-2752.

14. Loh KP, Abdallah M, Shieh MS, et al. Use of inpatient palliative care services in patients with advanced cancer receiving critical care therapies. J Natl Compr Canc Netw 2018;16:1055-1064.

15. Bamias A, Tzannis K, Harshman LC, et al. Impact of contemporary patterns of chemotherapy utilization on survival in patients with advanced cancer of the urinary tract: a Retrospective International Study of Invasive/ Advanced Cancer of the Urothelium (RISC). Ann Oncol 2018;29:361-369.

16. Necchi A, Sonpavde G, Lo Vullo S, et al. Nomogram-based prediction of overall survival in patients with metastatic urothelial carcinoma receiving first-line platinum-based chemotherapy: Retrospective International Study of Invasive/Advanced Cancer of the Urothelium (RISC). Eur Urol 2017;71:281-289.

17. Agency for Healthcare Research and Quality. HCUP Overview. Available at: https://www.hcup-us.ahrq.gov/overview.jsp. Accessed July 20, 2018.

18. Loh KP, Kansagra A, Shieh MS, et al. Predictors of in-hospital mortality in patients with metastatic cancer receiving specific critical care therapies. J Natl Compr Canc Netw 2016;14:979-987.

19. Deyo RA, Cherkin DC, Ciol MA. Adapting a clinical comorbidity index for use with ICD-9-CM administrative databases. J Clin Epidemiol 1992;45: 613-619.

20. Preisser F, Mazzone E, Nazzani S, et al. Comparison of perioperative outcomes between cytoreductive radical prostatectomy and radical prostatectomy for nonmetastatic prostate cancer. Eur Urol 2018;74 693-696.

21. Wadhwa $V$, Mehta D, Jobanputra $Y$, et al. Healthcare utilization and costs associated with gastroparesis. World J Gastroenterol 2017;23: 4428-4436.
22. Feder S, Tate J, Womack J, et al. Validation of the diagnostic code for palliative care in patients hospitalized with heart failure within the Department of Veterans Affairs [abstract]. J Pain Symptom Manage 2017;53 438-439.

23. Hua M, Li G, Clancy C, et al. Validation of the V66.7 code for palliative care consultation in a single academic medical center. J Palliat Med 2017;20: 372-377.

24. Mulvey CL, Smith TJ, Gourin CG. Use of inpatient palliative care services in patients with metastatic incurable head and neck cancer. Head Neck 2016;38:355-363.

25. Mazzone E, Nazzani S, Preisser F, et al. Partial nephrectomy seems to confer a survival benefit relative to radical nephrectomy in metastatic renal cell carcinoma. Cancer Epidemiol 2018;56:118-125

26. Abdollah F, Sammon JD, Majumder K, et al. Racial disparities in end-of-life care among patients with prostate cancer: a population-based study. J Natl Compr Canc Netw 2015;13:1131-1138.

27. Reyes-Gibby CC, Anderson KO, Shete S, et al. Early referral to supportive care specialists for symptom burden in lung cancer patients: a comparison of non-Hispanic whites, Hispanics, and non-Hispanic blacks. Cancer 2012 118:856-863.

28. Necchi A, Joseph RW, Loriot $Y$, et al. Atezolizumab in platinum-treated locally advanced or metastatic urothelial carcinoma: post-progression outcomes from the phase II IMvigor210 study. Ann Oncol 2017;28: 3044-3050.

29. Mazzone E, Preisser F, Nazzani S, et al. The effect of age and comorbidities on early postoperative complications after radical cystectomy: a contemporary population-based analysis. J Geriatr Oncol 2019;10:623-631.

30. Lee EW, Wei LJ, Amato DA, et al. Cox-type regression analysis for large numbers of small groups of correlated failure time observations. In: Klein JP, Goel PK, eds. Survival Analysis: State of the Art. Nato Science (Series E: Applied Sciences), vol. 211. Dordrecht, the Netherlands: Springer; 1992: 237-247.

31. Szekendi MK, Vaughn J, Lal A, et al. The prevalence of inpatients at 33 U.S. hospitals appropriate for and receiving referral to palliative care. J Palliat Med 2016;19:360-372. 